\title{
STATE AND EVENT ESTIMATION FOR REGIME-SWITCHING SYSTEMS UNDER IRREGULAR AND RANDOM SAMPLING SCHEMES*
}

\author{
WEI FENG ${ }^{\dagger}$ AND LEYI WANG ${ }^{\ddagger}$
}

\begin{abstract}
Estimation of states and events in randomly switching systems is studied under irregular and random sampling schemes. Probabilistic characterization of observability is presented under various sampling schemes and regime-switching processes. The characterization is derived on the basis of our recent results on sampling complexity for system observability. Observer design and algorithms are developed.
\end{abstract}

1. Introduction. This paper investigates estimation of states and events in randomly switching systems under various sampling schemes. The problems are typically studied under the names of regime-switching systems, hybrid systems, discrete-event systems, etc. Typically, such systems involve communication channels whose power and bandwidth limitations make it desirable to reduce resource consumption in sampling and quantization. It was shown in $[29,30]$ that traditional periodic sampling is inefficient in utility of such resources. More efficient sampling/quantization schemes introduced in $[29,30]$ lead naturally to irregular sampling (also known as non-uniform or non-periodic sampling). Irregular and random sampling may occur also due to event triggered sampling [3, 22] or communication uncertainty and interruptions [9].

When a system switches its dynamics, it introduces an event which is itself a dynamic process whose state space is a finite set and its state also needs to be estimated. State estimation of linear dynamic systems is a traditional topic that has been well studied [14]. Independently, observability of events has been studied extensively in discrete event systems $[15,18]$. References $[25,26]$ contain more recent studies on observability of sampled systems. Joint identification of states and events has been studied in hybrid systems [20,25]. Studies on fundamental properties of non-uniform sampling remain an active area of research, see [6] and the references therein for some recent work in this area. Some related results on identification, state estimation, and fault detection using binary or quantized outputs can be found in [13, 26, 31, 32, 35].

This paper studies some fundamental issues in joint estimation of states and events when the events are stochastic processes. The main issues are: What are the conditions for observability? How many sampling points are needed to ensure

\footnotetext{
*Dedicated to Professor Hanfu Chen on the occasion of his 75th birthday.

${ }^{\dagger}$ College of Information Science and Engineering, Shandong Agricultural University, China, Email: fwsnakefa@126.com

$\ddagger$ Department of Electrical and Computer Engineering, Wayne State University, Detroit, MI 48202, E-mail: lywang@ece.eng.wayne.edu.
} 
observability? What is the probabilistic description of observability? Our exploration of these topics utilizes some fundamental results from our recent work on minimum sampling density for state observability [33, 34]. Our recent work [29, 30] deals with deterministic regime-switching processes. This paper is focused on events that are stochastic processes.

The paper is organized into the following sections. Section 2 develops the basic framework in which estimation of states and events will be carried out. Sampling schemes and event processes are described. The PWM-based sampling scheme is discussed as a benchmark choice of the constant density irregular sampling schemes. Although joint observability and our estimation schemes do not rely on this specific scheme, it has distinctive advantages of using only one-bit transmission in communications and hence is very efficient in reducing communication resource consumption. Section 3 presents results on sampling complexity for state estimation of observable subsystems. Probabilistic characterization of joint observability is discussed under two event processes. The first case involves renewal processes, and the second Markov chains. Section 4 studies state observability of regime-switching systems in which subsystems are unobservable. In this case, regime switching helps enhancement of observability. Observer algorithms are derived. Joint state and event estimation is presented in Section 5. Finally, Section 6 summarizes findings of this paper and points out several open issues along the direction of this paper.

\section{Preliminaries.}

2.1. Systems. Suppose that $\gamma(t)$ is a discrete event process taking values in $\mathcal{M}=\{1,2, \ldots, m\}$, which represents regime switching in system dynamics ${ }^{1}$. Since the system input is irrelevant in state and event estimation problems here, we consider a linear regime-switching system without input

$$
\left\{\begin{array}{l}
\dot{x}(t)=A_{\gamma(t)} x(t) \\
y(t)=C_{\gamma(t)} x(t),
\end{array} \quad t \geq 0\right.
$$

where $A_{i} \in \mathbb{R}^{n \times n}$ and $C_{i} \in \mathbb{R}^{1 \times n}$ for each $i \in \mathcal{M}, x(t) \in \mathbb{R}^{n}$ is the continuous state, and $y(t) \in \mathbb{R}$ is the system output. In this paper, $x(t)$ is called "state" and $\gamma(t)$ "event". $M^{\prime}$ will denote the transpose of a matrix or vector $M$.

This paper assumes a two-time-scale scheme for the sampling times and regime switching (or system jumping) times; see Figure 1. Event switching occurs at $\tau_{k}$, $k=1,2, \ldots$ with the sojourn time (also known as the staying or holding time at a given subsystem) $s_{k}=\tau_{k}-\tau_{k-1}\left(\tau_{0}=0\right)$. Sampling of the output occurs at $t_{i}$,

\footnotetext{
${ }^{1}$ In this paper, $\gamma(t)$ represents the sequence of subsystems, rather than the switching from one subsystem to another. In the typical discrete event literature, the event is a switching between two subsystems.
} 
$i=1,2, \ldots$. The event $\gamma(t)$ remains a constant $\gamma_{k}$ during $t \in\left[\tau_{k}, \tau_{k+1}\right)$. As a result, $\gamma(t)$ becomes a discrete-time process $\gamma_{k}=\gamma\left(\tau_{k}\right)$. Depending on applications, we may need to estimate only the state or estimate jointly the state and event. However, the regime switching time $\tau_{k}$, which is random and its occurrence is unknown in advance, is assumed to be observed when it occurs. In other words, this paper does not deal with estimation of the regime switching time. In the subsequent development, the expression $\left[0, \tau_{1}\right)$ should be understood as a random interval.

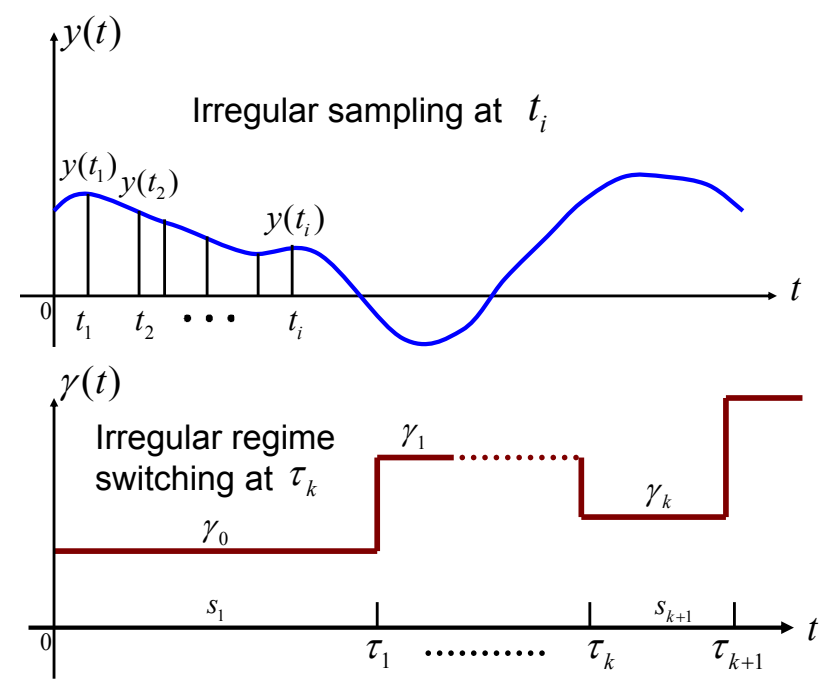

FIG. 1. The two-time-scale formulation. The regime switching occurs at a slower pace than the sampling speed. As a result, in between each regime switching, there can be many sampling points. Both $\tau_{k}$ and $t_{i}$ occur irregularly or randomly.

The output of the system is sampled at a set of $N$ time instances $\mathcal{T}_{N}=\left\{t_{i} \geq\right.$ $0, i=1, \ldots, N\}$, generating the set of observations $\mathcal{Y}_{N}=\left\{y\left(t_{i}\right), i=1, \ldots, N\right\}$. The observation data may be further divided by the time blocks: $\mathcal{Y}_{N}^{k}=\left\{y\left(t_{i}\right) \in \mathcal{Y}_{N}, t_{i} \in\right.$ $\left.\left[\tau_{k}, \tau_{k+1}\right)\right\}, k=0, \ldots, L-1$ with $t_{N} \in\left[\tau_{L-1}, \tau_{L}\right)$. The data in the set $\mathcal{Y}_{N}^{k}$ will be called "intra-block" data, and data across different blocks will be termed as "inter-block" data.

For $k=0, \ldots, L-1$, joint observability deals with reconstruction of the state $x(t)$ and event $\left\{\gamma_{k}, k=0, \ldots, L-1\right\}$ from the output observations $\mathcal{Y}_{N}$. Since $x(t)$ can be derived from $x(0)$ when the event sequence $\left\{\gamma_{k}, k=0, \ldots, L-1\right\}$ is identified, the state and event estimation problem is equivalent to reconstruction of the initial state $x(0)$ and $\left\{\gamma_{k}, k=0, \ldots, L-1\right\}$ from $\mathcal{Y}_{N}$.

2.2. Sampling Schemes. This paper will consider two possible irregular sampling schemes: (1) Constant-Density Irregular Sampling (CDIS) Scheme: In this scheme, the sampling times are irregular, but the sampling density $N / T$ (the number 
$N$ of samples in a time interval $T$ ) is fixed. This is most typically represented by PWM-based sampling Schemes. (2) Random Sampling (RS) Scheme: In this scheme, the sampling times are generated by a random process.

2.2.1. CDIS Schemes. To reduce communication resources, we introduce the following PWM-based (Pulse Width Modulation) observation scheme. This is a special case of threshold control in generating sampling sequences [29, 30, 33, 34]. This scheme allows a one-bit observation sequence that can drastically reduce communication resources. While this scheme can control the number of sample points in a given time interval, the sampling time is irregular.

Suppose that a signal $y(t)$ is bounded in its value by $y_{\min } \leq y(t) \leq y_{\max }$ and in its rate by $|\dot{y}(t)| \leq r$. A PWM carrier $h(t)$ is a periodic signal of period $\iota$ with lower value $h_{\min } \leq y_{\min }$ and upper value $h_{\max } \geq y_{\max }$, defined in one period by

$$
h(t)=\left\{\begin{array}{cc}
\frac{h_{\max }-h_{\min }}{\iota_{1}} t+h_{\min } ; & 0 \leq t<\iota_{1}, \\
0 ; & \iota_{1} \leq t<\iota,
\end{array}\right.
$$

where $\iota_{1}<\left(h_{\max }-h_{\min }\right) / r$, which guarantees that the rate of the carrier is higher than the rate of the signal. Note that if $\iota<\left(h_{\max }-h_{\min }\right) / r$, we may take $\iota_{1}=\iota$ and the carrier becomes the standard sawtooth waveform. This modulation signal will generate precisely one switching time $t_{i}$ in each period when $y(t)-h(t)$ switches its sign from positive to negative; see Figure 2. The known value $h\left(t_{i}\right)$ becomes the observed value of $y\left(t_{i}\right)$ although the time $t_{i} \in[(i-1) \iota, i \iota)$ is irregular. Due to communication uncertainties and discrepancies in clock synchronization, perfect synchronization and noise-free observations are not realistic. These effects are represented by noisy observations on $y$.

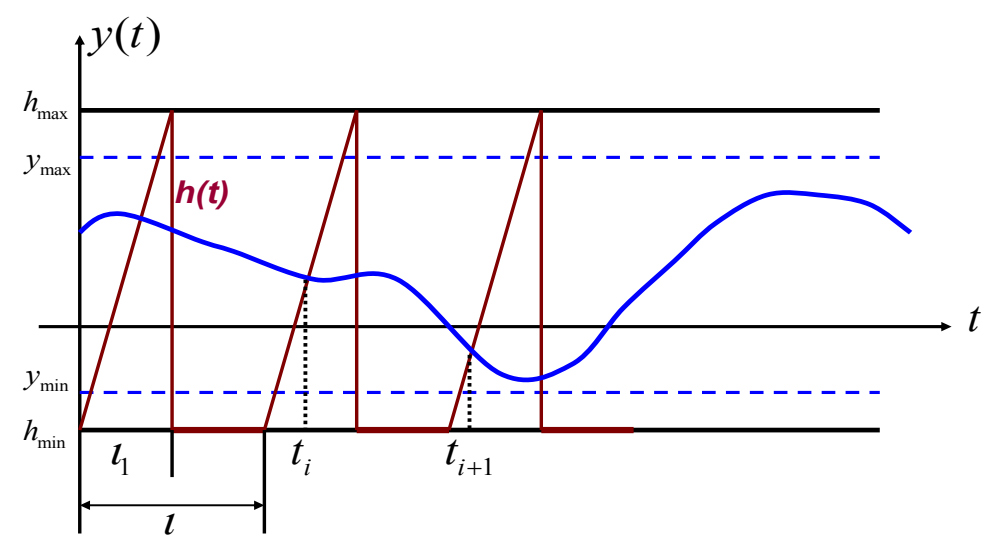

FIG. 2. PWM-based sampling

When this modulation scheme is used in communications, the modulation signal 
$h(t)$ is synchronized in both the sending end and the receiving end. The channel transmits a bit value "1" at the time of switching and does not transmit otherwise, generating an observation sequence $y\left(t_{i}\right), i=1,2, \ldots$ It is noted that for any given time interval $[0, T)$, the number $N_{0}$ of observation sample points is $\lfloor T / \iota\rfloor \leq N_{0} \leq$ $\lfloor T / \iota\rfloor+1$, and hence can be easily controlled by $\iota$. However, the actual sampling time sequence $\left\{t_{i}\right\}$ is irregular. $\delta=\lfloor T / \iota\rfloor$ will be interpreted as the data flow rate or bandwidth for observations. Such a sampling scheme will be called Constant Density Irregular Sampling (CDIS) of Rate $\delta$.

2.2.2. RS Schemes. When the sampling times are random, the number $N(T)$ of samples in the time interval $[0, T)$ becomes a counting process.

Assumption 1. The sampling process is a Poisson process of rate $\eta$, that is, the inter-sampling time $T_{i}=t_{i}-t_{i-1}$ is independent and exponentially distributed of rate $\eta$. Consequently, the counting process $N(T)$ has the probability distribution

$$
\mathbb{P}\{N(T)=k\}=\frac{(\eta T)^{k}}{k !} e^{-\eta T}, \quad k=0,1,2, \cdots,
$$

where $\mathbb{P}$ is the probability and $\eta$ is a positive constant, representing the average sampling rate.

2.3. Regime-Switching Time Sequences. The regime switching occurs randomly. We consider two types of event processes: Renewal Processes and Markov Chains.

2.3.1. Renewal Processes. Suppose that $k(t)$, the $k$ th regime switching, is a renewal process: $\{k(t), t \geq 0\}$ is a nonnegative integer-valued process where the time interval $s_{k}$ between the $(k-1)$ th event and the $k$ th event is positive, independent and identically distributed. The accumulative distribution function of $s_{k}$ is

$$
F(T)=\mathbb{P}\left\{s_{k} \leq T\right\}
$$

In this case, the event process will be termed as a renewal process of distribution $F(\cdot)$.

The most common renewal process is the Poisson process with parameter $\lambda$ whose inter-occurrence distribution is exponential

$$
F(T)=1-e^{-\lambda T}, T \geq 0 .
$$

This process will be called a Poisson process of parameter $\lambda$.

2.3.2. Markov Chains. Suppose that $\left\{\left(\gamma(t), \mathcal{F}_{t}\right), t \geq 0\right\}$ in $(2.1)$ is a continuous time homogeneous Markov chain with the state space $\mathcal{M}=\{1,2, \cdots, m\}$, transition rate matrix $Q=\left[q_{i j}\right]_{m \times m}$, and the initial distribution

$$
\mathbb{P}\{\gamma(0)=i\}=p_{i}, \quad i \in \mathcal{M} .
$$


Here, (i) $\left\{\mathcal{F}_{t}, t \geq 0\right\}$ is a right continuous filtration with respect to a given complete probability space $(\Omega, \mathcal{F}, \mathbb{P})$ and is augmented by all null sets in $\mathbb{P}$-completion of $\mathcal{F}$; (ii) $p_{i} \geq 0, i \in \mathcal{M}$, and $\sum_{i=1}^{m} p_{i}=1$; (iii) $q_{i j}$ is determined by

$$
\mathbb{P}\{\gamma(t+h)=j \mid \gamma(t)=i\}= \begin{cases}q_{i j} h+o(h), & i \neq j, \\ 1+q_{i i} h+o(h), & i=j,\end{cases}
$$

and $0 \leq q_{i}:=-q_{i i}=\sum_{j \neq i} q_{i j}$ for any $i \in \mathcal{M}$.

It is standard that the sojourn time $s_{k}$ of a homogeneous Markov chain is independent and exponentially distributed

$$
\mathbb{P}\left\{s_{k} \leq T \mid \gamma\left(\tau_{k-1}\right)=i\right\}=\left\{\begin{array}{ll}
1-e^{-q_{i} T}, & T \geq 0, \\
0, & T<0
\end{array} \quad i \in \mathcal{M} .\right.
$$

This event process will be called a Markov chain with generator $Q=\left[q_{i j}\right]$.

2.4. Estimation Problems. Depending on practical considerations, we may study the following two estimation problems.

(1) State Estimation (SE): In the SE problem, the event $\gamma_{k}$ is directly observed. Only $x(0)$ needs to be estimated. While state estimation problems are classical problems, SE under CDIS or RS schemes and regime-switching frameworks are new. This paper will also present results of state estimation of unobservable subsystems. In this case, regime switching is necessary and helpful in achieving state observability.

(2) Joint Estimation of State and Event (JE): In the JE problem, we must estimate both $x(0)$ and $\gamma_{k}$.

Definition 1. Suppose $t_{N}<\tau_{1}$. Namely, all output observations are contained in one block. (i) The system (2.1) is said to be $N$-sample state observable if $x(0)$ can be uniquely determined from any values $\mathcal{Y}_{N}$ on $\mathcal{T}_{N}$. (ii) The system (2.1) is said to be $N$-sample jointly observable if $x(0)$ and $\gamma_{0}$ can be uniquely determined from any values $\mathcal{Y}_{N}$ on $\mathcal{T}_{N}$.

3. State Estimation and Sampling Complexity for Observable Subsystems. We start with a review of certain basic relationships from $[29,30,33]$ that characterize sampling complexity for state and event estimation under irregular sampling. In this section, all subsystems are observable. Unobservable subsystems will be considered in Section 4.

Assumption 2. $\left(A_{i}, C_{i}\right), i \in \mathcal{M}$, are observable.

3.1. Basic Relationships. Suppose $A_{i}$ has eigenvalues $\lambda_{p}^{i}=\sigma_{p}^{i} \pm \mathrm{j} \omega_{p}^{i}, p=$ $1, \ldots, n$. Denote $\omega^{i}=\max _{p=1, \ldots, n}\left|\omega_{p}^{i}\right|$ and

$$
\omega=\max _{i=1, \ldots, m} \omega^{i} .
$$


$\omega^{i}$ may be interpreted as the "bandwidth" of the $i$ th subsystem. Then $\omega$ is the "bandwidth" of the entire system.

For generic system matrices $A$ and $C$, under $N$ sampling times $\left\{t_{i}, i=1, \ldots, N\right\}$, we have $y\left(t_{i}\right)=C e^{A t_{i}} x(0), i=1, \ldots, N$, which can be written as

$$
Y=M x(0), \quad Y=\left[\begin{array}{c}
y\left(t_{1}\right) \\
\vdots \\
y\left(t_{N}\right)
\end{array}\right], \quad M=\left[\begin{array}{c}
C e^{A t_{1}} \\
\vdots \\
C e^{A t_{N}}
\end{array}\right]
$$

We express $e^{A t}$ in terms of the matrices $I, A, A^{2}, \cdots, A^{n-1}$ (the Cayley-Hamilton Theorem), $e^{A t}=\alpha_{1}(t) I+\alpha_{2}(t) A+\cdots+\alpha_{n}(t) A^{n-1}$, where $\alpha(t)=\left[\alpha_{1}(t), \ldots, \alpha_{n}(t)\right]^{\prime}$ can be solved by the Lagrange-Hermite interpolation [11]. Suppose $A$ has $l$ distinct eigenvalues $\lambda_{i}, i=1, \ldots, l$ of multiplicity $m_{i}$, respectively. Here, $\sum_{i=1}^{l} m_{i}=n$. Define the $n$ modes of the $A$ matrix by

$$
\xi(t)=\left[e^{\lambda_{1} t}, \cdots, \frac{t^{m_{1}-1}}{\left(m_{1}-1\right) !} e^{\lambda_{1} t}, \cdots, e^{\lambda_{l} t}, \cdots, \frac{t^{m_{l}-1}}{\left(m_{l}-1\right) !} e^{\lambda_{l} t}\right]^{\prime},
$$

whose $n$ components are linearly independent. For any given $t>0$, the characteristic polynomial of $A t$ is $c_{A t}(z)=\prod_{i=1}^{l}\left(z-\lambda_{i} t\right)^{m_{i}}$. By [11, Section 6.1.14, pp. 390], there is a polynomial $r(z)=\sum_{p=0}^{n-1} c_{p+1} z^{p}$, which satisfies the interpolation conditions:

$$
\left.\frac{d^{j} r(z)}{d z^{j}}\right|_{z=\lambda_{i} t}=\left.\frac{d^{j} e^{z}}{d z^{j}}\right|_{z=\lambda_{i} t}=e^{\lambda_{i} t}, j=0, \cdots, m_{i}-1, i=1, \cdots, l .
$$

The coefficients $c_{p}(t), p=1 \cdots, n$, depending on $t$, are uniquely determined by (3.4). $r(z)$ is said to interpolate $e^{z}$ and its derivatives at the roots of $c_{A t}(z)$.

Let $\alpha_{p}(t)=c_{p}(t) t^{p-1}, p=1, \ldots, n$. Then, (3.4) can be rewritten as

$$
\Lambda^{\prime} \alpha(t)=\xi(t)
$$

where the $n \times n$ matrix $\Lambda^{\prime}$ depends on $\lambda_{i}, i=1, \ldots, l$ and their multiplicities. $\Lambda$ is invertible due to the uniqueness of solutions of (3.4) (see [11, pp. 390]). From the proof of $[11$, Theorem $6.2 .9(\mathrm{a})]$, one has

$$
e^{A t}=r(A t)=\alpha_{1}(t) I+\alpha_{2}(t) A+\cdots+\alpha_{n}(t) A^{n-1} .
$$

For any given $x(0), y(t)=C e^{A t} x(0)$ is a linear combination of the modes of $A$. As a result, it belongs to the class of exponential polynomials: for any $t \in[0, T]$ and $v=\left[v_{1,1}, \ldots, v_{1, m_{1}}, \ldots, v_{l, 1}, \ldots, v_{l, m_{l}}\right]^{\prime} \in \mathbb{C}^{n}$, let

$$
g(t)=\sum_{i=1}^{l} \sum_{j=1}^{m_{i}} v_{i, j} \frac{t^{j-1}}{(j-1) !} e^{\lambda_{i} t}
$$

Recall that a nonlinear function $g(t)$ is said to be non-trivial if $g(t) \not \equiv 0$. In reference to $\xi(t), g(t)=\xi^{\prime}(t) v$. Since the elements of $\xi(t)$ are linearly independent, for any $v \neq 0, g$ is non-trivial. 
3.2. Sampling Complexity of State Estimation under CDIS Schemes.

We first cite a result from [33] which forms a basis for our analysis on sampling complexity.

Lemma 1. [33] Consider $\xi(t)$ in (3.3). Suppose that $0<t_{1}<\cdots<t_{N}<T$. The matrix

$$
\Xi=\left[\begin{array}{c}
\xi^{\prime}\left(t_{1}\right) \\
\vdots \\
\xi^{\prime}\left(t_{N}\right)
\end{array}\right]
$$

is full rank if $N>2(n-1)+\frac{T \omega}{\pi}$.

From (3.6), we have

$$
C e^{A t}=\left[\alpha_{1}(t), \cdots, \alpha_{n}(t)\right]\left[\begin{array}{c}
C \\
C A \\
\vdots \\
C A^{n-1}
\end{array}\right]=\alpha^{\prime}(t) W_{o}
$$

where $W_{o}$ is the observability matrix of $(A, C)$.

For any $N$ sampling times $t_{i} \in[0, T), i=1,2, \cdots, N$, define

$$
\Gamma=\left[\begin{array}{c}
\alpha^{\prime}\left(t_{1}\right) \\
\vdots \\
\alpha^{\prime}\left(t_{N}\right)
\end{array}\right]
$$

Then,

$$
M=\left[\begin{array}{c}
C e^{A t_{1}} \\
\vdots \\
C e^{A t_{N}}
\end{array}\right]=\Gamma W_{o}
$$

The following key lemma on the number of zeros of exponential polynomials can be derived from [4, Theorem 3.2.47].

Lemma 2. [4] The number $N_{T}$ of zeros in $[0, T)$ of a non-trivial exponential polynomial $g$ defined in (3.7) is bounded by $N_{T} \leq 2(n-1)+\frac{T \omega}{\pi}$.

Lemмa 3. [30] (1) If $N>2(n-1)+\frac{T \omega}{\pi}$, then $\Gamma$ is full rank. (2) If, in addition, $(A, C)$ is observable, then $M$ is full rank.

THEOREM 1. [33] Suppose $\gamma_{0}$ is known and $\tau_{1}$ is the first switching time. Under Assumption 2, if the number $N$ of the sampling points in $\left[0, \tau_{1}\right)$ satisfies

$$
N>2(n-1)+\frac{\tau_{1} \omega}{\pi}
$$


then $x(0)$ can be uniquely determined from the sampled output values.

We now characterize state observability under randomly switching event processes. Note that since the event switching occurs randomly, state observability is a random entity. We will derive probabilistic and asymptotic characterization of this entity.

3.2.1. State Observability under Renewal Regime-Switching Processes. Under a CDIS scheme of rate $\delta$, the number of samples in an interval $[0, T)$ is $N(T)=$ $\lfloor\delta T\rfloor$, the largest integer not exceeding $\delta T$.

Lemma 4. Suppose that $\delta>\omega / \pi$. Under Assumption 2, if the first regimeswitching interval $\left[0, \tau_{1}\right)$ satisfies

$$
\tau_{1}>\frac{2(n-1)}{\delta-\frac{\omega}{\pi}},
$$

then $x(0)$ can be uniquely determined from the sampled values.

Proof. The observability condition (3.11) can be written as

$$
\delta=N / \tau_{1}>2(n-1) / \tau_{1}+\frac{\omega}{\pi} .
$$

Since $\delta>\omega / \pi,(3.13)$ can be expressed as a condition on $\tau_{1}$

$$
\tau_{1}>\frac{2(n-1)}{\delta-\frac{\omega}{\pi}} .
$$

Lemma 4 can be used to derive probabilistic descriptions of state observability. Suppose that the regime-switching is a renewal process of distribution $F(\cdot)$. For convenience of reference, denote the event of observable states by

$$
\Theta_{S}=\{x(0) \text { can be uniquely determined from the sampled values }\} \text {. }
$$

TheOREm 2. Suppose $\delta>\omega / \pi$. Under Assumption 2,

$$
\mathbb{P}\left\{\Theta_{S}\right\} \geq 1-F\left(\frac{2(n-1)}{\delta-\frac{\omega}{\pi}}\right) .
$$

Proof. By Lemma 4,

$$
\begin{aligned}
\mathbb{P}\left\{\Theta_{S}\right\} & \geq \mathbb{P}\left\{\tau_{1}>\frac{2(n-1)}{\delta-\frac{\omega}{\pi}}\right\} \\
& =1-\mathbb{P}\left\{\tau_{1} \leq \frac{2(n-1)}{\delta-\frac{\omega}{\pi}}\right\} \\
& =1-F\left(\frac{2(n-1)}{\delta-\frac{\omega}{\pi}}\right) .
\end{aligned}
$$


COROLlary 1. Suppose that the regime-switching process is a Poisson process with parameter $\lambda$. Then, (1)

$$
\mathbb{P}\left\{\Theta_{S}\right\} \geq e^{-\lambda \frac{2(n-1)}{\delta-\frac{\omega}{\pi}}}
$$

(2) If $\delta \geq \delta_{0}>\omega / \pi$, then

$$
\mathbb{P}\left\{\Theta_{S}\right\} \rightarrow 1 \text {, as } \lambda / \delta \rightarrow 0
$$

Proof.

(1) (3.16) follows directly from (2.4) and (3.15).

(2) Since

$$
e^{-\lambda \frac{2(n-1)}{\delta-\frac{\omega}{\pi}}}=e^{-\frac{\lambda}{\delta} \frac{2(n-1)}{1-\frac{\omega}{\delta \pi}}} \geq e^{-\frac{\lambda}{\delta} \frac{2(n-1)}{1-\frac{\omega}{\delta 0 \pi}}} \rightarrow 1, \text { as } \lambda / \delta \rightarrow 0
$$

and $\mathbb{P}\left\{\Theta_{S}\right\} \leq 1,(3.16)$ implies that $\mathbb{P}\left\{\Theta_{S}\right\} \rightarrow 1$, as $\lambda / \delta \rightarrow 0$.

\subsubsection{State Observability under Infrequently Switching Event Process-}

es. Suppose that the event $\gamma(t)$ ia a continuous-time Markov chain with a finite state space $\mathcal{M}=\{1, \ldots, m\}$ and generator $\varepsilon Q$. Let $p_{j}(t)=\mathbb{P}\{\gamma(t)=j\}, j=1, \ldots, m$ and $p(t)=\left[p_{1}(t), \ldots, p_{m}(t)\right]^{\prime}$. The process $\gamma(t)$ is governed by

$$
\frac{d p(t)}{d t}=p(t) \varepsilon Q
$$

where $Q=\left[q_{i j}\right] \in \mathbb{R}^{m \times m}$ is the generator satisfying $q_{i j} \geq 0$ for $i \neq j, \sum_{j=1}^{m} q_{i j}=0$ for each $i=1, \ldots, m$.

Here $\varepsilon$ is a small parameter that indicates a relatively "infrequently switching" Markov chain in relation to the sampling density $\delta$. For state observability, the relationship between $\varepsilon$ and $\delta$ is given by the following theorem. Denote $q_{\text {max }}=\max _{i=1, \ldots, m}\left|q_{i i}\right|$.

THEOREM 3. Suppose $\delta \geq \delta_{0}>\omega / \pi$.

(1) Under Assumption 2, if the event $\gamma(t)$ is a continuous-time Markov chain with generator $\varepsilon Q$ then

$$
\mathbb{P}\left\{\Theta_{S}\right\} \geq e^{-\varepsilon q_{\max } \frac{2(n-1)}{\delta-\frac{\omega}{\pi}}} .
$$

$$
\mathbb{P}\left\{\Theta_{S}\right\} \rightarrow 1, \text { as } \varepsilon / \delta \rightarrow 0
$$

Proof. (1) Under (3.18), the probability that no transition occurs in the time interval $\left[0, \tau_{1}\right)$ when $\gamma(0)=i$ is

$$
\begin{aligned}
P_{i}\left(\tau_{1}\right) & =\mathbb{P}\left\{\gamma(t)=i, \forall t \in\left(0, \tau_{1}\right) \mid \gamma(0)=i\right\} \\
& =e^{-\varepsilon q_{i i} \tau_{1}} \\
& \geq e^{-\varepsilon q_{\max } \tau_{1}} .
\end{aligned}
$$


(3.19) follows from

$$
\begin{aligned}
\mathbb{P}\left\{\Theta_{S}\right\} & \geq \max _{j=1, \ldots, m} P_{j}\left(\frac{2(n-1)}{\delta-\frac{\omega}{\pi}}\right) \\
& \geq e^{-\varepsilon q_{\max } \frac{2(n-1)}{\delta-\frac{\omega}{\pi}}} .
\end{aligned}
$$

(2) This follows from

$$
e^{-\varepsilon q_{\max } \frac{2(n-1)}{\delta-\frac{\omega}{\pi}}} \geq e^{-\frac{\varepsilon}{\delta} q_{\max } \frac{2(n-1)}{1-\frac{\omega}{\delta} \pi}} \rightarrow 1, \text { as } \varepsilon / \delta \rightarrow 0
$$

REMARK 1. $\varepsilon / \delta$ is the ratio of the regime-switching "frequency" and the sampling "frequency". This theorem indicates that if the sampling frequency is relatively higher than the regime switching frequency, state observability will be guaranteed with high probability.

3.3. State Observability under RS Schemes. We now consider the random sampling schemes. Unlike the case of CDIS schemes in which the sampling times are irregular but not random, here we are dealing with two random processes: The sampling times and regime switching. Interaction of these two processes will characterize the state observability.

\subsubsection{State Observability under Renewal Event Processes.}

Assumption 3. (a) $\left(A_{i}, C_{i}\right), i \in \mathcal{M}$, are observable. (b) The sampling process satisfies Assumption 1. (c) The regime switching process $\gamma(t)$ is a renewal process with distribution $F(\cdot)$ whose density function exists and is denoted by $f(\cdot)$. (d) The sampling process is independent of the regime switching process.

Denote

$$
\mu_{\tau}=\left\lfloor 2(n-1)+\frac{\tau \omega}{\pi}\right\rfloor
$$

and $N_{\tau}$ the number of samples in $[0, \tau)$. In particular, let $\tau_{1}$ denote the first switching time of the system.

THEOREM 4. Under Assumption 3, if $\gamma(0)=i$, then

$$
\mathbb{P}\left\{\Theta_{S}\right\} \geq 1-\int_{0}^{\infty} \sum_{k=0}^{\mu_{\tau}} \frac{(\eta \tau)^{k}}{k !} e^{-\eta \tau} f(\tau) d \tau
$$

Proof. First, we note that

$$
\mathbb{P}\left\{\Theta_{S}\right\} \geq \mathbb{P}\left\{N_{\tau_{1}}>\mu_{\tau_{1}} \mid \gamma(0)=i\right\}
$$


From the total probability formula, we have

$$
\begin{aligned}
& \mathbb{P}\left\{N_{\tau_{1}}>\mu_{\tau_{1}} \mid \gamma(0)=i\right\} \\
& =\int_{0}^{\infty} \mathbb{P}\left\{N_{\tau}>\mu_{\tau} \mid \gamma_{0}, \tau\right\} f(\tau) d \tau \\
& =\int_{0}^{\infty} \sum_{k=\mu_{\tau}+1}^{\infty} \frac{(\eta \tau)^{k}}{k !} e^{-\eta \tau} f(\tau) d \tau \\
& =\int_{0}^{\infty}\left(1-\sum_{k=0}^{\mu_{\tau}} \frac{(\eta \tau)^{k}}{k !} e^{-\eta \tau}\right) f(\tau) d \tau \\
& =1-\int_{0}^{\infty} \sum_{k=0}^{\mu_{\tau}} \frac{(\eta \tau)^{k}}{k !} e^{-\eta \tau} f(\tau) d \tau
\end{aligned}
$$

which implies (3.21).

COROLlary 2. Under the conditions of Theorem 4, if the renewal process is actually Poisson with parameter $\lambda$, then (1)

$$
\mathbb{P}\left\{\Theta_{S}\right\} \geq 1-\lambda \int_{0}^{\infty} \sum_{k=0}^{\mu_{\tau}} \frac{(\eta \tau)^{k}}{k !} e^{-(\eta+\lambda) \tau} d \tau
$$

$$
\lim _{\lambda / \eta \rightarrow 0} \mathbb{P}\left\{\Theta_{S}\right\}=1
$$

Proof.

(1) This follows by replacing $f(\cdot)$ with

$$
f(\tau)=\lambda e^{-\lambda \tau}
$$

(2) Let $\bar{\tau}=\eta \tau$. Then $\mu_{\tau}=\left\lfloor 2(n-1)+\frac{\bar{\tau} \omega}{\eta \pi}\right\rfloor:=\bar{\mu}_{\bar{\tau}}$.

$$
\int_{0}^{\infty} \sum_{k=0}^{\mu_{\tau}} \frac{(\eta \tau)^{k}}{k !} e^{-\eta \tau} \lambda e^{-\lambda \tau} d \tau=\int_{0}^{\infty} \sum_{k=0}^{\bar{\mu}_{\bar{\tau}}} \frac{(\bar{\tau})^{k}}{k !} e^{-\bar{\tau}} \frac{\lambda}{\eta} e^{-\frac{\lambda}{\eta} \bar{\tau}} d \bar{\tau}
$$

Since this integral is uniformly convergent with respect to $\lambda / \eta$,

$$
\lim _{\lambda / \eta \rightarrow 0} \int_{0}^{\infty} \sum_{k=0}^{\bar{\mu}_{\bar{\tau}}} \frac{(\bar{\tau})^{k}}{k !} e^{-\bar{\tau}} \frac{\lambda}{\eta} e^{-\frac{\lambda}{\eta} \bar{\tau}} d \bar{\tau}=\int_{0}^{\infty} \sum_{k=0}^{\bar{\mu}_{\bar{\tau}}} \frac{(\bar{\tau})^{k}}{k !} e^{-\bar{\tau}}\left(\lim _{\lambda / \eta \rightarrow 0} \frac{\lambda}{\eta} e^{-\frac{\lambda}{\eta} \bar{\tau}}\right) d \bar{\tau}=0
$$

This, together with (3.22) and the fact that $\mathbb{P}\left\{\Theta_{S}\right\} \leq 1$, implies (3.23).

REMARK 2. Similar to CDIS schemes, here $\lambda / \eta$ is the ratio of the regimeswitching "frequency" and the average sampling "frequency". This theorem indicates that if the average sampling frequency is relatively higher than the regime switching frequency, state observability will be guaranteed with high probability. 
THEOREM 5. Under the conditions of Theorem 4, if the renewal process is actually Poisson with parameter $\lambda$, then, for any integer $R \geq 0$,

$$
\begin{aligned}
\mathbb{P}\left\{\Theta_{S}\right\} \geq & -e^{-\frac{\lambda \pi}{\omega}(R+1)}+\frac{\lambda}{\lambda+\eta} \sum_{r=0}^{R} e^{-\frac{(\eta+\lambda) \pi}{\omega}(r+1)} \sum_{k=0}^{2 n-2+r} \frac{1}{k !}\left(\frac{\eta \pi(r+1)}{\omega}\right)^{k} \\
& -\frac{\lambda}{\lambda+\eta} \sum_{r=0}^{R} e^{-\frac{(\eta+\lambda) \pi}{\omega} r} \sum_{k=0}^{2 n-2+r} \frac{1}{k !}\left(\frac{\eta \pi r}{\omega}\right)^{k} \\
& -\frac{\lambda}{\eta} \sum_{r=0}^{R} e^{-\frac{(\eta+\lambda) \pi}{\omega}(r+1)} \sum_{k=0}^{2 n-2+r} \frac{\eta^{k+1}}{(\lambda+\eta)^{k+1}} \sum_{q=0}^{k-1} \frac{1}{q !}\left(\frac{(\lambda+\eta) \pi}{\omega}(r+1)\right)^{q} \\
& +\frac{\lambda}{\eta} \sum_{r=0}^{R} e^{-\frac{(\eta+\lambda) \pi}{\omega} r} \sum_{k=0}^{2 n-2+r} \frac{\eta^{k+1}}{(\lambda+\eta)^{k+1}} \sum_{q=0}^{k-1} \frac{1}{q !}\left(\frac{(\lambda+\eta) \pi}{\omega} r\right)^{q} .
\end{aligned}
$$

In particular, for $R=0$ we have

$$
\begin{aligned}
\mathbb{P}\left\{\Theta_{S}\right\} \geq & 1-e^{-\frac{\lambda \pi}{\omega}}+\frac{\lambda}{\lambda+\eta} e^{-\frac{(\eta+\lambda) \pi}{\omega}} \sum_{k=0}^{2 n-2} \frac{1}{k !}\left(\frac{\eta \pi}{\omega}\right)^{k} \\
& -\frac{\lambda}{\eta} e^{-\frac{(\eta+\lambda) \pi}{\omega}} \sum_{k=0}^{2 n-2}\left(\frac{\eta}{\lambda+\eta}\right)^{k+1} \sum_{q=0}^{k-1} \frac{1}{q !}\left(\frac{(\eta+\lambda) \pi}{\omega}\right)^{q} .
\end{aligned}
$$

Proof. By Corollary 2,

$$
\begin{aligned}
\mathbb{P}\left\{\Theta_{S}\right\} & \geq \lambda \int_{0}^{\infty} \sum_{k=\mu_{\tau}}^{+\infty} \frac{(\eta \tau)^{k}}{k !} e^{-(\eta+\lambda) \tau} d \tau \\
& =\lambda \sum_{r=0}^{+\infty} \int_{\frac{r \pi}{\omega}}^{\frac{(r+1) \pi}{\omega}} \sum_{k=2 n-1+r}^{+\infty} \frac{(\eta \tau)^{k}}{k !} e^{-(\eta+\lambda) \tau} d \tau .
\end{aligned}
$$

Thus, for any integer $R \geq 0$, we have

$$
\begin{aligned}
\mathbb{P}\left\{\Theta_{S}\right\} & >\lambda \sum_{r=0}^{R} \int_{\frac{r \pi}{\omega}}^{\frac{(r+1) \pi}{\omega}} \sum_{k=2 n-1+r}^{+\infty} \frac{(\eta \tau)^{k}}{k !} e^{-(\eta+\lambda) \tau} d \tau \\
& =\lambda \sum_{r=0}^{R} \int_{\frac{r \pi}{\omega}}^{\frac{(r+1) \pi}{\omega}}\left(1-\sum_{k=0}^{2 n-2+r} \frac{(\eta \tau)^{k}}{k !} e^{-\eta) \tau}\right) e^{-\lambda \tau} d \tau \\
& =\lambda \sum_{r=0}^{R} \int_{\frac{r \pi}{\omega}}^{\frac{(r+1) \pi}{\omega}} e^{-\lambda \tau} d \tau-\lambda \sum_{r=0}^{R} \sum_{k=0}^{2 n-2+r} \int_{\frac{r \pi}{\omega}}^{\frac{(r+1) \pi}{\omega}} \frac{(\eta \tau)^{k}}{k !} e^{-\eta \tau} e^{-\lambda \tau} d \tau .
\end{aligned}
$$

Let $\rho=\eta \tau$. Then, from the second summation term

$$
\frac{\lambda}{\eta} \sum_{r=0}^{R} \sum_{k=0}^{2 n-2+r} \int_{\frac{r \pi}{\omega}}^{\frac{(r+1) \pi}{\omega}} \frac{(\eta \tau)^{k}}{k !} e^{-\eta \tau} e^{-\lambda \tau} d \tau
$$


in $(3.27)$, we obtain

$$
\begin{aligned}
& \int_{\frac{\pi}{\omega} r}^{\frac{\pi}{\omega}(r+1)} \frac{(\eta \tau)^{k}}{k !} e^{-(\eta+\lambda) \tau} d \tau=\frac{1}{k !} \int_{\frac{\eta \pi}{\omega} r}^{\frac{\eta \pi}{\omega}(r+1)} \rho^{k} e^{-\left(1+\frac{\lambda}{\eta}\right) \rho} d \rho \\
= & -\frac{\eta}{(\lambda+\eta) k !}\left[\left(\frac{\eta \pi}{\omega}(r+1)\right)^{k} e^{-\left(1+\frac{\lambda}{\eta}\right) \frac{\eta \pi}{\omega}}-\left(\frac{\eta \pi}{\omega} r\right)^{k}\right] e^{-\left(1+\frac{\lambda}{\eta}\right) \frac{\eta \pi}{\omega} r} \\
& +\sum_{p=1}^{k} \frac{k(k-1) \cdots(k-p+1)}{k !\left(1+\frac{\lambda}{\eta}\right)^{p+1}}\left[\left(\frac{\eta \pi}{\omega}(r+1)\right)^{k-p} e^{-\left(1+\frac{\lambda}{\eta}\right) \frac{\eta \pi}{\omega}}\right. \\
& \left.-\left(\frac{\eta \pi}{\omega} r\right)^{k-p}\right] e^{-\left(1+\frac{\lambda}{\eta}\right) \frac{\eta \pi}{\omega} r} .
\end{aligned}
$$

Denote $k-p=q$. Then,

$$
\begin{aligned}
& \int_{\frac{\pi}{\omega} r}^{\frac{\pi}{\omega}(r+1)} \frac{(\eta \tau)^{k}}{k !} e^{-(\eta+\lambda) \tau} d \tau \\
= & -\frac{\eta e^{-\frac{(\lambda+\eta) \pi}{\omega} r}}{(\lambda+\eta) k !}\left[\left(\frac{\eta \pi}{\omega}(r+1)\right)^{k} e^{-\frac{(\lambda+\eta) \pi}{\omega}}-\left(\frac{\eta \pi}{\omega} r\right)^{k}\right] \\
+ & \frac{e^{-\frac{(\lambda+\eta) \pi}{\omega} r}}{\left(1+\frac{\lambda}{\eta}\right)^{k+1}} \sum_{q=0}^{k-1} \frac{1}{q !}\left[\left(\frac{(\lambda+\eta) \pi}{\omega}(r+1)\right)^{q} e^{-\frac{(\lambda+\eta) \pi}{\omega}}-\left(\frac{(\lambda+\eta) \pi}{\omega} r\right)^{q}\right] .
\end{aligned}
$$

Thus, we can derive the summation

$$
\frac{\lambda}{\eta} \sum_{r=0}^{R} \sum_{k=0}^{2 n-2+r} \int_{\frac{r \pi}{\omega}}^{\frac{(r+1) \pi}{\omega}} \frac{(\eta \tau)^{k}}{k !} e^{-\eta \tau} e^{-\lambda \tau} d \tau .
$$

By

$$
\sum_{r=0}^{R} \int_{\frac{r \pi}{\omega}}^{\frac{(r+1) \pi}{\omega}} e^{-\lambda \tau} d \tau=1-e^{\frac{\lambda(R+1) \pi}{\omega}}
$$

and (3.30) we obtain (3.24) and (3.25).

REMARK 3. Following the same arguments and the proof of Theorem 5, similar results can be readily obtained for Theorem 6, Theorem 14 and Corollary 5 below, respectively. For conciseness, these obvious results are omitted.

\subsubsection{State Observability under Markov Event Processes.}

Assumption 4. (a) $\left(A_{i}, C_{i}\right), i \in \mathcal{M}$, are observable. (b) The sampling process satisfies Assumption 1. (c) The regime switching process $\gamma(t)$ is a Markov chain with generator $Q$. (d) The sampling process is independent of the regime switching process.

Denote

$$
\mu_{\tau}=\left\lfloor 2(n-1)+\frac{\tau \omega}{\pi}\right\rfloor
$$


and $N_{\tau}$ the number of samples in $[0, \tau)$. In particular, let $\tau_{1}$ be the first switching time of the system. By (2.7), the sojourn time has distribution

$$
\begin{aligned}
F_{i}(\tau) & =\mathbb{P}\left\{\tau_{1} \leq \tau \mid \gamma(0)=i\right\} \\
& =1-e^{-q_{i} \tau}, \tau \geq 0,
\end{aligned}
$$

and its density function is

$$
f_{i}(\tau)=q_{i} e^{-q_{i} \tau}, \quad \tau \geq 0 .
$$

Theorem 6. Under Assumption 4,

(1) if $\gamma(0)=i$, then

$$
\mathbb{P}\left\{\Theta_{S}\right\} \geq 1-q_{i} \int_{0}^{\infty} \sum_{k=0}^{\mu_{\tau}} \frac{(\eta \tau)^{k}}{k !} e^{-\left(\eta+q_{i}\right) \tau} d \tau .
$$

$$
\lim _{q_{i} / \lambda \rightarrow 0} \mathbb{P}\left\{\Theta_{S}\right\}=1 .
$$

Proof. The proof is similar to those of Theorem 4 and Corollary 2.

Remark 4. Similar to CDIS schemes, here $q_{i} / \eta$ is the ratio of the regimeswitching "frequency" and the average sampling "frequency". This theorem indicates that if the average sampling frequency is much higher than the regime switching frequency, state observability will be guaranteed with high probability.

\section{State Estimation and Sampling Complexity for Unobservable Sub-} systems.

4.1. Enhancement of Observability by Regime Switching. We now remove the critical condition that all subsystems must be observable. The main idea is that although some subsystems may not be observable, by regime-switching, they may collaboratively provide observability for the initial state. In this section, we focus on state estimation. So, the event sequence is known when it occurs.

Suppose that the $i$ th subsystem has its observability matrix $W_{i}, i=1, \ldots, m$, which may not be full rank. Since the subsystems may not be observable, without regime switching the initial state $x(0)$ cannot be uniquely determined from the output observations.

Consider now the event sequence that contains at least one regime switching. Suppose that the involved subsystems are $\left(A_{\gamma_{0}}, C_{\gamma_{0}}\right)$ and $\left(A_{\gamma_{1}}, C_{\gamma_{1}}\right)$ with their observability matrices $W_{\gamma_{0}}$ and $W_{\gamma_{1}}$, respectively. Denote the composite matrix

$$
W=\left[\begin{array}{l}
W_{\gamma_{0}} \\
W_{\gamma_{1}}
\end{array}\right] .
$$


The numbers of samples are $N_{0}$ in the interval $\left[0, \tau_{1}\right)$ and $N_{1}$ in the interval $\left[\tau_{1}, \tau_{2}\right)$. The sojourn times are $s_{0}=\tau_{1}$ and $s_{1}=\tau_{2}-\tau_{1}$.

Assumption 5. (a) $W$ is full rank. (b) $N_{i}>2(n-1)+\frac{s_{i} \omega_{i}}{\pi}, i=0,1$.

TheOREM 7. Under Assumption 5, x(0) can be uniquely determined from the sampled output values.

REMARK 5. This theorem requires $W$ to be full rank, but not individual $W_{i}$. As a result, it deals with individually unobservable subsystems which are, however, collectively observable. In this sense, regime-switching can potentially improve state observability.

Proof. The observation equation is

$$
Y=M x(0)=\left[\begin{array}{c}
M_{\gamma_{0}} \\
M_{\gamma_{1}}
\end{array}\right] x(0)=\left[\begin{array}{c}
\Gamma_{\gamma_{0}} W_{\gamma_{0}} \\
\Gamma_{\gamma_{1}} W_{\gamma_{1}} e^{A_{\gamma_{0}} \tau_{1}}
\end{array}\right] x(0) .
$$

To show state observability, we only need to prove that $M$ is full column rank.

For any $\beta \in \mathbb{R}^{n}$, suppose $M \beta=0$. That is

$$
\Gamma_{\gamma_{0}} W_{\gamma_{0}} \beta=0, \Gamma_{\gamma_{1}} W_{\gamma_{1}} e^{A_{\gamma_{0}} \tau_{1}} \beta=0 .
$$

Denote $\beta_{1}=e^{A_{\gamma_{0}} \tau_{1}} \beta$. Now, $\Gamma_{\gamma_{0}} W_{\gamma_{0}} \beta=0$ are $N_{0}$ zeros of an exponential polynomial of $n$ modes. Similarly for $\Gamma_{\gamma_{1}} W_{\gamma_{1}} \beta_{1}=0$. Under Assumption 5 (b), by Lemma 2, we have

$$
W_{\gamma_{0}} \beta=0, W_{\gamma_{1}} \beta_{1}=0
$$

$W_{\gamma_{0}} \beta=0$ means that $\beta \in \operatorname{ker}\left(W_{\gamma_{0}}\right)$, the kernel of $W_{\gamma_{0}}$. By the Cayley-Hamilton Theorem,

$$
\operatorname{ker}\left(W_{\gamma_{0}}\right)=\operatorname{ker}\left(\left[\begin{array}{c}
C_{\gamma_{0}} \\
C_{\gamma_{0}} A_{\gamma_{0}} \\
C_{\gamma_{0}} A_{\gamma_{0}}^{2} \\
\vdots
\end{array}\right]\right)
$$

Hence, $\beta_{1}=e^{A_{\gamma_{0}} \tau_{1}} \beta \in \operatorname{ker}\left(W_{\gamma_{0}}\right)$. On the other hand, $W_{\gamma_{1}} \beta_{1}=0$ means $\beta_{1} \in$ $\operatorname{ker}\left(W_{\gamma_{1}}\right)$. As a result, $\beta_{1} \in \operatorname{ker}\left(W_{\gamma_{0}}\right) \cap \operatorname{ker}\left(W_{\gamma_{1}}\right)$.

By Assumption 5 (a), $W$ is full rank, which implies that $\operatorname{ker}\left(W_{\gamma_{0}}\right) \cap \operatorname{ker}\left(W_{\gamma_{1}}\right)=$ $\{0\}$. This implies $\beta_{1}=0$. It follows that $\beta=e^{-A_{\gamma_{1}} \tau_{1}} \beta_{1}=0$. Since $\beta$ is arbitrary, $M$ must be full column rank.

4.2. An Example. Consider a regime-switching system involving two subsystems

$$
A_{1}=\left[\begin{array}{cc}
0 & 1 \\
0 & -2
\end{array}\right], C_{1}=[0,1] ; A_{2}=\left[\begin{array}{cc}
1 & 0 \\
-0.3 & 0
\end{array}\right], C_{2}=[1,0]
$$


The corresponding observability matrices are

$$
W_{1}=\left[\begin{array}{cc}
0 & 1 \\
0 & -2
\end{array}\right] \text { and } W_{2}=\left[\begin{array}{cc}
1 & 0 \\
1 & 0
\end{array}\right]
$$

They are singular, indicate that both subsystems are unobservable. However,

$$
W=\left[\begin{array}{l}
W_{1} \\
W_{2}
\end{array}\right]=\left[\begin{array}{cc}
0 & 1 \\
0 & -2 \\
1 & 0 \\
1 & 0
\end{array}\right]
$$

is full column rank. Hence, the two subsystems are collectively observable.

The true initial state is $x(0)=[10,10]^{\prime}$. The system is run under the subsystem 1 with $N$ randomly sampled points in the time interval $[0, T)$, then is switched to the subsystem 2 with another $N$ randomly sampled points in $[T, 2 T)$. In the following simulation, $T=5$ second. The system output is corrupted by measurement noises of i.i.d. normal random variables of mean 0 and variance 25 .

For $N=20$, the state estimation algorithm was executed 100 times and the estimation errors are recorded. The estimation errors have an average 0.1157 and variance 0.0115 .

For $N=100$, the state estimation algorithm was executed 100 times and the estimation errors are recorded. The estimation errors have an average 0.0830 and variance 0.0051 .

For $N=200$, the state estimation algorithm was executed 100 times and the estimation errors are recorded. The estimation errors have an average 0.0348 and variance 0.0028 .

4.3. Probabilistic Characterization. We now establish $\mathbb{P}\left\{\Theta_{S}\right\}$ under unobservable subsystems.

Assumption 6. (a) The matrix $W$ in Assumption 5 is full rank. (b) The sampling process satisfies Assumption 1. (c) The regime switching process $\gamma(t)$ is a renewal process with distribution $F(\cdot)$ whose density function exists and is denoted by $f(\cdot)$.

(d) The sampling process is independent of the regime switching process.

Denote

$$
\mu_{\tau}=\left\lfloor 2(n-1)+\frac{\tau \omega}{\pi}\right\rfloor
$$

TheOREm 8. Under Assumption 6,

$$
\mathbb{P}\left\{\Theta_{S}\right\} \geq\left(1-\int_{0}^{\infty} \sum_{k=0}^{\mu_{\tau}} \frac{(\eta \tau)^{k}}{k !} e^{-\eta \tau} f(\tau) d \tau\right)^{2}
$$


Proof. Suppose that Assumption 5 (a) holds true. By Theorem 7, to achieve state observability, a sufficient condition is that $N_{i}>2(n-1)+\frac{s_{i} \omega_{i}}{\pi}, i=0,1$. This implies that

$$
\begin{aligned}
\mathbb{P}\left\{\Theta_{S}\right\} & \geq \mathbb{P}\left\{N_{0}>2(n-1)+\frac{s_{0} \omega_{0}}{\pi} \text { and } N_{1}>2(n-1)+\frac{s_{1} \omega_{1}}{\pi}\right\} \\
& =\mathbb{P}\left\{N_{0}>2(n-1)+\frac{s_{0} \omega_{0}}{\pi}\right\} \mathbb{P}\left\{N_{1}>2(n-1)+\frac{s_{1} \omega_{1}}{\pi}\right\}
\end{aligned}
$$

since $N_{0}, s_{0}, N_{1}, s_{1}$ are mutually independent.

On the other hand, by Theorem 4 , for $i=0,1$

$$
\mathbb{P}\left\{N_{i}>2(n-1)+\frac{s_{i} \omega_{i}}{\pi}\right\} \geq 1-\int_{0}^{\infty} \sum_{k=0}^{\mu_{\tau}} \frac{(\eta \tau)^{k}}{k !} e^{-\eta \tau} f(\tau) d \tau
$$

and (4.1) follows.

4.4. Recursive Algorithms under Inter-Block Observations. When subsystems are unobservable, regime switching is utilized to enhance observability. Consequently, state estimation involves data from different blocks. Suppose that for the $k$ th block $\left[\tau_{k}, \tau_{k+1}\right)$, the event is $\gamma_{k}$. For inter-block information integration, the goal is to use all data before $\tau_{k}$ to estimate $x_{k}=x\left(\tau_{k}\right)$. As a result,this is a prediction problem. In principle, if $\widehat{x}_{k}$ is an estimate of $x_{k}$ based on data before $\tau_{k}$, then the one-block prediction for $x_{k+1}$ will be denoted by $\widehat{x}_{k+1}^{p}=e^{A_{\gamma_{k}} T_{k}} \widehat{x}_{k}$.

We aim to update $\widehat{x}_{k+1}^{p}$ to $\widehat{x}_{k+1}$ by using the additional observations in $\left[\tau_{k}, \tau_{k+1}\right)$. Assume that $N_{k}$ samples of the output occur at $\tau_{k}<t_{1}^{k}<\cdots<t_{N_{k}}^{k}<\tau_{k+1}$ with the noise-corrupted observation values $z_{1}^{k}, \ldots, z_{N_{k}}^{k}$. To relate these data to $x_{k+1}$, we denote

$$
\Phi_{k+1}=\left[\begin{array}{c}
C_{\gamma_{k}} e^{A_{\gamma_{k}}\left(t_{1}^{k}-\tau_{k+1}\right)} \\
\vdots \\
C_{\gamma_{k}} e^{A_{\gamma_{k}}\left(t_{N_{k}}^{k}-\tau_{k+1}\right)}
\end{array}\right], Z_{k+1}=\left[\begin{array}{c}
z_{1}^{k} \\
\vdots \\
z_{N_{k}}^{k}
\end{array}\right]
$$

for the data. On the other hand, all past data before $\tau_{k}$ will be collectively written in the observation equation as $\bar{Z}_{k}=\bar{\Phi}_{k} x_{k}+\bar{D}_{k}$. It follows that

$$
\widehat{x}_{k}=\left(\bar{\Phi}_{k}^{\prime} \bar{\Phi}_{k}\right)^{-1} \bar{\Phi}_{k}^{\prime} \bar{Z}_{k}
$$

We would like to derive an iteration for $\widehat{x}_{k}$.

Although $\widehat{x}_{k}$ in (4.2) appears to be in a LS form, the standard recursive LS algorithm is not applicable. To understand this, note that in the observation equation $\bar{Z}_{k+1}=\bar{\Phi}_{k+1} x_{k+1}+\bar{D}_{k+1}, \bar{\Phi}_{k+1}=\left[\begin{array}{c}\bar{\Phi}_{k} e^{-A_{\gamma_{k}} T_{k}} \\ \Phi_{k}\end{array}\right]$. In other words, it is not merely an addition of one block to the matrix. 
Let $Q_{k}=e^{A_{\gamma_{k}} T_{k}}$. Then $\bar{\Phi}_{k+1}=\left[\begin{array}{c}\bar{\Phi}_{k} Q_{k}^{-1} \\ \Phi^{k+1}\end{array}\right], \bar{Z}_{k+1}=\left[\begin{array}{c}\bar{Z}_{k} \\ Z_{k+1}\end{array}\right]$. In the following derivation, let $\bar{P}_{k}=\left(\bar{\Phi}_{k}^{\prime} \bar{\Phi}_{k}\right)^{-1}, \bar{K}_{k}=Q_{k}^{-1} \bar{P}_{k} \Phi_{k+1}^{\prime}$.

THEOREM 9. $\widehat{x}_{k}$ in (4.2) can be updated recursively by

$$
\begin{aligned}
\widehat{x}_{k+1} & =Q_{k+1}\left(I-\bar{K}_{k+1} \Phi_{k+1} Q_{k+1}\right) \widehat{x}_{k}+\bar{P}_{k+1} \Phi_{k+1}^{\prime} Z_{k+1} \\
\bar{K}_{k+1} & =\bar{P}_{k} Q_{k+1}^{\prime} \Phi_{k+1}^{\prime}\left(1+\Phi_{k+1} Q_{k+1} \bar{P}_{k} Q_{k+1}^{\prime} \Phi_{k+1}^{\prime}\right)^{-1} \\
\bar{P}_{k+1} & =Q_{k+1}\left(I-\bar{K}_{k+1} \Phi_{k+1} Q_{k+1}\right) \bar{P}_{k} \Phi_{k+1}^{\prime} .
\end{aligned}
$$

Proof. Since $\bar{\Phi}_{k+1}=\left[\begin{array}{c}\bar{\Phi}_{k} Q_{k+1}^{-1} \\ \Phi_{k+1}\end{array}\right]$, by the matrix inversion lemma,

$$
\begin{aligned}
\bar{P}_{k+1}=\left(\bar{\Phi}_{k+1}^{\prime} \bar{\Phi}_{k+1}\right)^{-1} \\
=\left(\left(Q_{k+1}^{-1}\right)^{\prime} \bar{\Phi}_{k}^{\prime} \bar{\Phi}_{k} Q_{k+1}^{-1}+\Phi_{k+1}^{\prime} \Phi_{k+1}\right)^{-1} \\
=Q_{k+1}\left(\bar{P}_{k}^{-1}+Q_{k+1}^{\prime} \Phi_{k+1}^{\prime} \Phi_{k+1} Q_{k+1}\right)^{-1} Q_{k+1}^{\prime} \\
=Q_{k+1}\left(\bar{P}_{k}-\bar{P}_{k} Q_{k+1}^{\prime} \Phi_{k+1}^{\prime}\right. \\
\left.\quad \times\left(1+\Phi_{k+1} Q_{k+1} \bar{P}_{k} Q_{k+1}^{\prime} \Phi_{k+1}^{\prime}\right)^{-1} \Phi_{k+1} Q_{k+1} \bar{P}_{k}\right) Q_{k+1}^{\prime} .
\end{aligned}
$$

Let

$$
\begin{aligned}
& \bar{K}_{k+1}:=Q_{k+1}^{-1} \bar{P}_{k+1} \Phi_{k+1}^{\prime} \\
& =\left(\bar{P}_{k}-\bar{P}_{k} Q_{k+1}^{\prime} \Phi_{k+1}^{\prime}\left(1+\Phi_{k+1} Q_{k+1} \bar{P}_{k} Q_{k+1}^{\prime} \Phi_{k+1}^{\prime}\right)^{-1}\right. \\
& \left.\quad \times \Phi_{k+1} Q_{k+1} \bar{P}_{k}\right) Q_{k+1}^{\prime} \Phi_{k+1}^{\prime} \\
& =\bar{P}_{k} Q_{k+1}^{\prime} \Phi_{k+1}^{\prime}\left(1+\Phi_{k+1} Q_{k+1} \bar{P}_{k} Q_{k+1}^{\prime} \Phi_{k+1}^{\prime}\right)^{-1}
\end{aligned}
$$

which implies $\bar{P}_{k+1}=Q_{k+1}\left(I-\bar{K}_{k+1} \Phi_{k+1} Q_{k+1}\right) \bar{P}_{k} Q_{k+1}^{\prime}$. Moreover,

$$
\begin{aligned}
\bar{\Phi}_{k+1}^{\prime} \bar{Z}_{k+1} & =\left[\left(Q_{k+1}^{-1}\right)^{\prime} \bar{\Phi}_{k}^{\prime}, \Phi_{k+1}^{\prime}\right]\left[\begin{array}{c}
\bar{Z}_{k} \\
Z_{k+1}
\end{array}\right] \\
& =\left(Q_{k+1}^{-1}\right)^{\prime} \bar{\Phi}_{k}^{\prime} \bar{Z}_{k}+\Phi_{k+1}^{\prime} Z_{k+1} .
\end{aligned}
$$

Now,

$$
\begin{aligned}
\widehat{x}_{k+1}= & \bar{P}_{k+1} \bar{\Phi}_{k+1}^{\prime} \bar{Z}_{k+1} \\
= & Q_{k+1}\left(I-\bar{K}_{k+1} \Phi_{k+1} Q_{k+1}\right) \bar{P}_{k} Q_{k+1}^{\prime}\left(Q_{k+1}^{-1}\right)^{\prime} \bar{\Phi}_{k}^{\prime} \bar{Z}_{k} \\
& +\bar{P}_{k+1} \Phi_{k+1}^{\prime} Z_{k+1} \\
= & Q_{k+1}\left(I-\bar{K}_{k+1} \Phi_{k+1} Q_{k+1}\right) \widehat{x}_{k} \\
& +\bar{P}_{k+1} \Phi_{k+1}^{\prime} Z_{k+1} \\
= & Q_{k+1}\left(I-\bar{K}_{k+1} \Phi_{k+1} Q_{k+1}\right) \widehat{x}_{k}+\bar{P}_{k+1} \Phi_{k+1}^{\prime} Z_{k+1} .
\end{aligned}
$$

5. Joint Estimation and Sampling Complexity. When $\gamma_{k}$ is unknown, we would like to estimate simultaneously $x\left(\tau_{k}\right)$ and $\gamma_{k}$ in the time interval $\left[\tau_{k}, \tau_{k+1}\right)$. Since $\gamma_{k}$ is a constant in this interval, without loss of generality we may assume $k=0$ 
and focus on joint estimation of $x(0)$ and $\gamma_{0}$ in $\left[0, \tau_{1}\right)$, where $\tau_{1}$ is the first switching time.

Assumption 7. (a) $\left(A_{i}, C_{i}\right), i \in \mathcal{M}$, are observable; (b) For any $i \neq j, A_{i}$ and $A_{j}$ do not share any common eigenvalues.

\subsection{Sampling Complexity.}

TheOREm 10. [29, 30] Under Assumption 7, if the number $N$ of the samples in $\left[0, \tau_{1}\right)$ satisfies

$$
N>2(2 n-1)+\frac{\tau_{1} \omega}{\pi},
$$

then both $x(0)$ and $\gamma_{0}$ can be uniquely determined from the sampled values.

REMARK 6 . This theorem states that if within a time block of length $\tau_{1}$ before the regime switching occurs, the number of output samples exceeds $2(2 n-1)+\frac{\tau_{1} \omega}{\pi}$, regardless the actual sampling times, the system is jointly observable.

Under the conditions of Theorem 10, we estimate $x(0)$ and $\gamma_{0}$ by seeking the unique solution $\gamma_{0}$ and $x(0)$ to

$$
Y=M_{\gamma_{0}} x(0) .
$$

For $i=1, \ldots, m$, we first calculate $m$ possible initial states

$$
\widehat{x}^{i}(0)=\left(M_{i}^{\prime} M_{i}\right)^{-1} M_{i}^{\prime} Y .
$$

Note that (5.3) can always be calculated since $\left(A_{i}, C_{i}\right)$ is observable and $N>2(2 n-$ 1) $+\frac{\tau_{1} \omega}{\pi}$, which imply that $M_{i}$ is full column rank. By Theorem 10, only one $i$ for which

$$
Y=M_{i} \widehat{x}^{i}(0)
$$

Consequently, $\gamma_{0}$ can be estimated by a simple elimination process: $\gamma_{0}=j$ is ruled out if $Y \neq M_{j} \widehat{x}^{j}(0)$ (even though $M_{j}^{\prime} Y=M_{j}^{\prime} M_{j} \widehat{x}^{j}(0)$ ).

REMARK 7. Naturally, one may also consider the problem of pure event estimation: $x(0)$ is known, but $\gamma_{0}$ is unknown. Although $x(0)$ is known in this case, to distinguish the events, it seems that $2(2 n-1)+\frac{\tau_{1} \omega}{\pi}$ sampling points may still be needed, the same number as the case when both $x(0)$ and $\gamma_{0}$ are unknown. We shall explain this by the following derivations.

From

$$
C_{\gamma_{0}} e^{A_{\gamma_{0}} t}=\alpha_{\gamma_{0}}^{\prime}(t) W_{\gamma_{0}}
$$

Now,

$$
M_{\gamma_{0}}=\left[\begin{array}{c}
C_{\gamma_{0}} e^{A_{\gamma_{0}} t_{1}} \\
\vdots \\
C_{\gamma_{0}} e^{A_{\gamma_{0}} t_{N}}
\end{array}\right]=\Gamma_{\gamma_{0}} W_{\gamma_{0}}, \Gamma_{\gamma_{0}}=\left[\begin{array}{c}
\alpha_{\gamma_{0}}^{\prime}\left(t_{1}\right) \\
\vdots \\
\alpha_{\gamma_{0}}^{\prime}\left(t_{N}\right)
\end{array}\right]
$$




$$
\Gamma_{\gamma_{0}}=\Xi_{\gamma_{0}} \Lambda_{\gamma_{0}}^{-1} ; M_{\gamma_{0}}=\Xi_{\gamma_{0}} \Lambda_{\gamma_{0}}^{-1} W_{\gamma_{0}} .
$$

Let $j \neq \gamma_{0}$ be another regime such that they produce the same sampled values

$$
\Xi_{\gamma_{0}} \Lambda_{\gamma_{0}}^{-1} W_{\gamma_{0}} x(0)=\Xi_{j} \Lambda_{j}^{-1} W_{j} x(0),
$$

which implies

$$
\left(\Xi_{\gamma_{0}} \Lambda_{\gamma_{0}}^{-1} W_{\gamma_{0}}-\Xi_{j} \Lambda_{j}^{-1} W_{j}\right) x(0)=0 .
$$

The rows of the left-hand side are the $N$ values of an exponential polynomial that contains $2 n$ modes. By Lemma 1 , since $N>2(2 n-1)+\frac{\tau_{1} \omega}{\pi}$, the polynomial is trivial, ie., $x(0)=0$. This contradicts to the assumption $x(0) \neq 0$. As a result, $j=\gamma_{0}$.

In the above analysis, the information on $x(0)$ does not seem to reduce sampling complexity for estimating the event. On the other hand, in practical applications, when $x(0)$ is known, the output trajectories from the $m$ subsystems can be directly calculated. As a result, an elimination algorithm can be used to delete the subsystems when their outputs are not equal to the sampled values until only one is left.

The following results are similar to the state estimation cases. Hence the proofs will be omitted.

\subsection{Joint Observability under CDIS Schemes.}

\subsubsection{Joint Observability under Renewal Regime-Switching Processes.}

Under a CDIS scheme of rate $\delta$, the number of samples in an interval of length $\tau$ is $N(\tau)=\lfloor\delta \tau\rfloor$.

Corollary 3. Suppose $\delta>\omega / \pi$. Under Assumption 7, if the first regimeswitching interval $\left[0, \tau_{1}\right)$ satisfies

$$
\tau_{1}>\frac{2(2 n-1)}{\delta-\frac{\omega}{\pi}}
$$

then both $x(0)$ and $\gamma_{0}$ can be uniquely determined from the sampled values.

Suppose that the regime-switching process is a renewal process with distribution $F(\cdot)$. Denote

$\Theta_{J}=\left\{\right.$ both $x(0)$ and $\gamma_{0}$ can be uniquely determined from the sampled values $\}$.

TheOREm 11. Suppose $\delta \geq \delta_{0}>\omega / \pi$. Under Assumption 7,

$$
\mathbb{P}\left\{\Theta_{J}\right\} \geq 1-F\left(\frac{2(2 n-1)}{\delta-\frac{\omega}{\pi}}\right) .
$$

Corollary 4. Suppose that the regime-switching is a Poisson process with parameter $\lambda$. Then, (1)

$$
\mathbb{P}\left\{\Theta_{J}\right\} \geq e^{-\lambda \frac{2(2 n-1)}{\delta-\frac{\omega}{\pi}}} .
$$


(2)

$$
\mathbb{P}\left\{\Theta_{J}\right\} \rightarrow 1, \text { as } \lambda / \delta \rightarrow 0
$$

\subsubsection{Joint Observability under Infrequently Switching Event Process-} es.

THEOREM 12. Suppose $\delta \geq \delta_{0}>\omega / \pi$.

(1) Under Assumption 7, if the event $\gamma(t)$ is a continuous-time Markov chain with generator $\varepsilon Q$ then

$$
\mathbb{P}\left\{\Theta_{J}\right\} \geq e^{-\varepsilon q_{\max } \frac{2(2 n-1)}{\delta-\frac{\omega}{\pi}}}
$$

(2)

$$
\mathbb{P}\left\{\Theta_{J}\right\} \rightarrow 1, \text { as } \varepsilon / \delta \rightarrow 0
$$

5.3. Joint Observability under RS Schemes. We now consider the random sampling schemes. Denote

$$
\mu_{\tau}=\left\lfloor 2(2 n-1)+\frac{\tau \omega}{\pi}\right\rfloor,
$$

and $N_{\tau}$ the number of samples in $[0, \tau)$. In particular, let $\tau_{1}$ be the first switching time of the system.

\subsubsection{Joint Observability under Renewal Event Processes.}

Assumption 8. (a) $\left(A_{i}, C_{i}\right), i \in \mathcal{M}$, are observable. (b) The sampling process satisfies Assumption 1. (c) The regime switching process $\gamma(t)$ is a renewal process with distribution $F(\cdot)$ whose density function exists and is denoted by $f(\cdot)$. (d) The sampling process is independent of the regime switching process.

TheOrem 13. Under Assumption 8,

$$
\mathbb{P}\left\{\Theta_{S}\right\} \geq 1-\int_{0}^{\infty} \sum_{k=0}^{\mu_{\tau}} \frac{(\eta \tau)^{k}}{k !} e^{-\eta \tau} f(\tau) d \tau .
$$

COROLlary 5. Under the conditions of Theorem 13, if the renewal process is actually Poisson with parameter $\lambda$, then (1)

$$
\mathbb{P}\left\{\Theta_{S}\right\} \geq 1-\lambda \int_{0}^{\infty} \sum_{k=0}^{\mu_{\tau}} \frac{(\eta \tau)^{k}}{k !} e^{-(\eta+\lambda) \tau} d \tau .
$$

(2)

$$
\lim _{\lambda / \eta \rightarrow 0} \mathbb{P}\left\{\Theta_{S}\right\}=1
$$




\subsubsection{Joint Observability under Markov Event Processes.}

Assumption 9. (a) $\left(A_{i}, C_{i}\right), i \in \mathcal{M}$, are observable. (b) The sampling process satisfies Assumption 1. (c) The regime switching process $\gamma(t)$ is a Markov chain with generator $Q$. (d) The sampling process is independent of the regime switching process.

By (2.7), the sojourn time has distribution

$$
F_{i}(\tau)=1-e^{-q_{i} \tau}, \tau \geq 0,
$$

and its density function is

$$
f_{i}(\tau)=q_{i} e^{-q_{i} \tau}, \tau \geq 0
$$

TheOREm 14. Under Assumption 9, (1)

$$
\mathbb{P}\left\{\Theta_{S}\right\} \geq 1-\max _{i=1, \ldots, m} q_{i} \int_{0}^{\infty} \sum_{k=0}^{\mu_{\tau}} \frac{(\eta \tau)^{k}}{k !} e^{-\left(\eta+q_{i}\right) \tau} d \tau .
$$

(2)

$$
\lim _{q_{i} / \lambda \rightarrow 0} \mathbb{P}\left\{\Theta_{S}\right\}=1 .
$$

6. Concluding Remarks. This paper provides probabilistic characterization of joint observability when the events are stochastic processes. The findings of this paper show that the relative rate of sampling and regime switching is the key to ensure high probability of observability. In the case of renewal processes and infrequently switching Markov chains, such a relationship is explicitly derived. There are many open problems along the direction of this paper. Studies of networked systems are the primary motivation for this study. Ideas of this paper can be directly applied to networked systems but detailed treatments of network topologies will be of substantial interests in this pursuit.

\section{REFERENCES}

[1] S. Ammar And J.-C. Vivalda, On the preservation of observability under sampling, Systems and Control Letters, 52(2004), pp. 7-15.

[2] J. AN AND D.M. YuAn, Complete convergence of weighted sums for $\rho^{*}$-mixing sequence of random variables, Statistics and Probability Letters, 78(2008), pp. 1466-1472.

[3] B.B. Astrom and B. Bernhardsson, Comparison of periodic and event based sampling for first-order stochastic systems, Proc. of the 14th IFAC World Congress, Beijing, pp. 301306, 1999.

[4] C.A. Berenstein And R. Gay, Complex Analysis and Special Topics in Harmonic Analysis, Springer, New York, 1995.

[5] Y. S. Chow And H. Teicher, Probability Theory, Springer-Verlag, New York, 3rd Ed., 1997. 
[6] F. Ding, L. QIU, AND T.W. CHEN, Reconstruction of continuous-time systems from their non-uniformly sampled discrete-time systems, Automatica, 45:2(2009), pp. 324-332.

[7] S.N. Ethier And T.G. Kurtz, Markov Processes: Characterization and Convergence, J. Wiley, New York, NY, 1986.

[8] J.-P. Gauthier And I. KupKA, Observability and observers for nonlinear systems, SIAM J. Control Optim., 32:4(1994), pp. 975-994.

[9] L. Guo, Convergence and logarithm laws of self-tuning regulators, Automatica, 31:3(1995), pp. 435-450.

[10] J.P. Hespanha And A.S. Morse. Stability of Switched Systems with Average Dwell-Time, Proc. of the 38th IEEE Conference on Decision and Control, pp. 2655-2660, 1999.

[11] R.A. Horn and C.R. Johnson, Topics in Matrix Analysis, Cambridge University Press, 1991.

[12] S. KAn, G. YIN, AND L. Y. WANG, System identification: Regime switching, unmodeled dynamics, and binary sensors, Nonliear Analysis, 71(2009), e1328-e1336.

[13] X. Koutsoukos, Estimation of hybrid systems using discrete sensors, Proc. of the 42nd IEEE Conference on Decision and Control, pp. 155-160, 2003.

[14] B.C. KuO, Digital Control Systems, 2nd. Ed., Oxford University Press, 1995.

[15] R. Kumar, V. K. Garg, and S.I. Marcus, On Controllability and Observability of Discrete Event Dynamic Systems, System and Control Letters, 17(1991), pp. 157-168.

[16] H.J. Kushner And G. Yin, Stochastic Approximation and Recursive Algorithms and Applications, 2nd Ed., Springer-Verlag, New York, 2003.

[17] S. LANG, Linear Algebra, 3rd Ed., Springe, 1987.

[18] F. Lin And W.M. Wonham, On observability of discrete-event systems, Information Sciences, 44:3(1988), pp. 173-198.

[19] E. Lukacs, Stochastic Convergence, Academic Press, New York, 1975.

[20] M. Mariton, Jump Linear Systems in Automatic Control, Marcel Dekker, New York, 1990.

[21] A. Papoulis and S.U. Pillai, Probability, Random Variables, and Stochastic Processes, 4th Ed., McGraw Hill, 2002.

[22] N. Persson And F. Gustafsson, Event based sampling with application to vibration analysis in pneumatic tire, in ICASSP 2001.

[23] C.L. Phillips And H.T. Nagle, Digital Control System Analysis and Design, 3rd Ed., Prentice Hall, 1994.

[24] J.G. Proakis and D.K. Manolakis, Digital Signal Processing, 4th Ed., Prentice Hall, 2006.

[25] J. RAISCH, Controllability and observability of simple hybrid control systems-FDLTI plants with symbolic measurements and quantized control inputs, CONTROL'94, pp.595-600, 1994.

[26] J. Sur AND B.E. PADEN, State observer for linear time-invariant systems with quantized output, ASME J. Dynamic Systems, Measurement, and Control, Vol.120, 1998.

[27] H.M. Taylor And S. Karlin, An Introduction to Stochastic Modeling, 3rd Ed., Academic Press, 1998.

[28] Sergey Utev and Magda Peligrad, Maximal Inequalities and an Invariance Principle for a Class of Weakly Dependent Random Variables, Journal of Theoretical Probability, 16:1(2003), pp. 101-115.

[29] L.Y. Wang, W. Feng, And G. YIN, Joint State and Event Observers for Irregularly Sampled Switching Systems, Proc. of the 16th IFAC Symposium on System Identification (SYSID 2012), Brussels, July 11-13, 2012.

[30] L.Y. WANG, W. FENG, AND G. Yin, Joint state and event observers for linear switching systems under irregular sampling, provisionally accepted by Automatica.

[31] L.Y. WANG, G.H. XU, AND G. YIN, State Reconstruction for Linear Time-Invariant Systems with Binary-Valued Output Observations, Systems and Control Letters, 57(2008), pp. 958- 
963.

[32] L.Y. WANG, G. Yin, J.F. ZhANG, AND Y.L. ZhaO, System identification with quantized observations, Birkhäuser, Boston, 2010.

[33] Le Yi Wang, Chanying Li, George Yin, Lei Guo, and Chengzhong Xu, State Observability and Observers of Linear-Time-Invariant Systems under Irregular-Sampling and Sensor Limitations, IEEE Transactions on Automatic Control, 56:11(2011), pp. 2639-2654.

[34] C. Li, L.Y. Wang, G. Yin, L. Guo, and C.-Z. Xu, Irregular Sampling, Active Observability, and Convergence Rates of State Observers for Systems with Binary-Valued Observations, Proc. of Joint 48th IEEE Conference on Decision and Control and 28th Chinese Control Conference, Shanghai, P.R. China, 2009.

[35] L.Y. WANG, J.F. Zhang, AND G. Yin, System identification using binary sensors, IEEE Transactions on Automatic Control, 48(2003), pp. 1892-1907.

[36] G. YIN, S. KAN, L.Y. WANG, AND C.Z. XU, Identification of systems with regime switching and unmodeled dynamics, IEEE Transactions on Automatic Control, 54:1(2009), pp. 34-47.

[37] G. Yin And Q. Zhang, Discrete-Time Markov Chains: Two-Time-Scale Methods and Applications, Springer, New York, 2005. 
